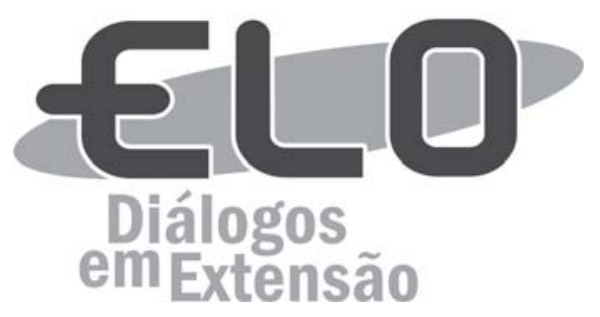

\title{
Os tesouros escondidos da terra revelados a partir da metodologia das instalações artístico-pedagógicas
}

\author{
Nancy Aidé Cardona Casas ${ }^{1}$, Bruna Carolina da Silva Goulart ${ }^{2}$, Vanessa Schiavon Lopes ${ }^{3}$, Fernanda \\ Bazilia da Costa Lemes ${ }^{4}$, André Luiz Miranda ${ }^{5}$, Laércio dos Anjos Benjaminn ${ }^{6}$, Irene Maria Cardoso ${ }^{7}$
}

\begin{abstract}
Resumo: A Troca de Saberes é um evento realizado na Universidade Federal de Viçosa, que busca o diálogo entre saberes científico e popular. Durante o evento foi realizada a Instalação Artístico-Pedagógica denominada "Os tesouros escondidos da roça". Diversos elementos foram organizados no espaço de forma harmônica com a intencionalidade pedagógica de favorecer o diálogo entre os participantes. A instalação foi planejada a partir de um "círculo de cultura", e contou com a participação de dois professores e 16 estudantes da graduação e pós-graduação da UFV. O objetivo foi gerar visibilidade aos processos, organismos e pessoas que vivem na roça e que têm sido historicamente invisibilizados. Durante a Troca de Saberes, 25 pessoas participaram, entre elas duas crianças e uma jovem surda. A participação efetiva de todos, a troca de conhecimentos e saberes e o reconhecimento dos tesouros da roça foram os principais resultados deste trabalho, importantes para o entendimento dos sistemas de produção.
\end{abstract}

Palavras-chave: Agroecologia. Solos. Educação. Metodologia Participativa. Pesquisa Participativa.

Área Temática: Agroecologia.

\section{The hidden treasures of the earth revealed from the methodology of the pedagogical artistic facilities}

Abstract: The Exchange of Knowledge is an event held at the Federal University of Viçosa and seeks to bridge the gap between scientific and popular knowledge. During the Artistic-Pedagogical Installation the "The Hidden Treasures of the Roça" was held. Several elements were presented in a beautiful and harmonious way with the pedagogical intention of provoking dialogue among the participants. The installation was planned from a "circle of culture" and was attended by two professors and 16 undergraduate and graduate students from UFV. The objective was to give visibility to processes, organisms and people who live in the countryside and who have been historically unacknowledged. During the Exchange of Knowledge, 20 people participated, among them two children and a deafyoung woman. The effective participation of all, the exchange of knowledge, and the recognition of the treasures discovered in the field are the main focuses of this article and are important for the understanding of the production systems.

Keywords: Agroecology. Soils. Education. Participatory methodology. Participatory Research.

\footnotetext{
${ }^{1}$ Pós-Graduanda em Solos e Nutrição de Plantas - Universidade Federal de Viçosa, UFV-MG, nacardonc@gmail.com Av. Peter Henry Rolfs s/n - Campus Universitário - CEP: 36570-900 - Viçosa, MG. Tel: (31) 99102-6251

${ }^{2}$ Pós-Graduanda em Agroecologia - Universidade Federal de Viçosa, UFV-MG.

${ }^{3}$ Pós-Graduanda em Solos e Nutrição de Plantas - Universidade Federal de Viçosa, UFV-MG.

${ }^{4}$ Graduanda em Agronomia - Universidade Federal de Viçosa, UFV-MG.

${ }^{5}$ Pós-Graduando em Solos e Nutrição de Plantas - Universidade Federal de Viçosa, UFV-MG.

${ }^{6}$ Ph.D., Professor - Universidade Federal de Viçosa, UFV-MG.

${ }^{7}$ Ph.D., Professora - Universidade Federal de Viçosa, UFV-MG.
} 


\section{Los tesoros ocultos del campo revelados a partir de la metodología de las instalaciones artistico pedagógicas}

Resumen: El Intercambio de Saberes es un evento realizado en la Universidad Federal de Viçosa y que busca el diálogo entre los saberes científico y popular. Durante el evento se realizó la Instalación ArtísticoPedagógica denominada "Los tesoros escondidos del campo". Diversos elementos fueron organizados en el espacio de forma bella y armónica con la intencionalidad pedagógica de favorecer el diálogo entre los participantes. La instalación fue planificada a partir de un "círculo de cultura", y contó con la participación de dos profesores y 16 estudiantes de pregrado y posgrado de la UFV. El objetivo fue dar visibilidad a procesos, organismos y personas que viven en el campo y que han sido históricamente invisibilizados. Durante el Intercambio de Saberes, 20 personas participaron, entre ellas dos niños y una joven sorda. La participación efectiva de todos, el intercambio de conocimientos y saberes y el reconocimiento de los tesoros del campo fueron los principales resultados de este trabajo, importantes para el entendimiento de los sistemas de producción.

Palabras clave: Agroecología. Suelos. Educación. Metodología Participativa. Investigación Participativa.

\section{Introdução}

A Troca de Saberes é um evento realizado anualmente na Universidade Federal de Viçosa que objetiva promover a ecologia de saberes, através da ressignificação, reelaboração e articulação entre os conhecimentos produzidos na universidade e na sociedade. Os saberes de diversos grupos sociais têm sido invisibilizados pelo modelo de desenvolvimento atual, que não reconhece os saberes populares, uniformiza culturas e homogeniza modos de pensar (SANTOS; BARBOSA; KÖLLN, 2013). Na busca de outro modelo de desenvolvimento, a ecologia de saberes promove espaços de diálogo entre os saberes populares e científicos, a partir do pressuposto que ambos são importantes e complementares (SANTOS; BARBOSA; KÖLLN , 2013).

Desde 2010, na Trocas de Saberes, para a promoção dos diálogos de saberes, a metodologia denominada instalações artístico-pedagógicas tem sido utilizada. Com esta metodologia, criam-se cenários com o objetivo de promover processos educativos a partir do diálogo sobre experiências vividas, o que, no contexto acadêmico, incluem as pesquisas realizadas (VILLAR et al., 2011). A instalação artístico-pedagógica traz consigo a beleza do ensinar e aprender, a partir da ética e da estética, consideradas dimensões fundamentais do ato educativo (FREIRE, 2011; CONTE et al., 2014).

Em 2018, na X Troca de Saberes, a Instalação Artístico-Pedagógica intitulada "Os tesouros escondidos da roça" foi organizada com o objetivo de ressignificar e reconhecer processos, seres e saberes invisibilizados no meio rural, denominado em Minas Gerais de "roças".

\section{Objetivos}

Sistematizar a experiência obtida com a Instalação Artístico-Pedagógica "Os tesouros escondidos da roça" para apontar as lições aprendidas com o uso da metodologia e gerar visibilidade aos processos, organismos e pessoas que vivem na roça e que têm sido historicamente invisibilizados.

\section{Metodologia}

A Instalação Artístico-Pedagógica "Os tesouros escondidos da roça" foi organizada de forma coletiva e interdisciplinar por 16 estudantes e dois professores (uma professora) dos programas de Pós-graduação de Solos e Nutrição de Plantas e de Agroecologia da Universidade Federal de Viçosa (UFV) e do programa internacional de pesquisa denominado "Benefícios da natureza nas fronteiras agroflorestais: conectando atores, estratégias, biodiversidade funcional e serviços dos ecossistemas" (FOREFRONT). Este programa é uma parceria da UFV com a Universidade de Wageningen (Holanda), a Universidade Nacional e o Colégio da Fronteira Sul (ambos do México). As pesquisas dos participantes são desenvolvidas com agricultores(as) familiares da região da Zona da Mata mineira, alguns participantes da Instalação.

A partir de duas reuniões, nas quais foram utilizadas metodologias participativas, os facilitadores puderam sonhar e planejar coletivamente a Instalação. Para isto, utilizou-se o "círculo de cultura", que se inicia com uma pergunta geradora e cada participante apresenta uma palavra ou elemento que responda tal pergunta (FREIRE, 2005; GADOTTI, 2000). As respostas de todos são sintetizadas de 
forma a responder coletivamente à pergunta. Assim, o círculo de cultura propicia a troca de experiências de forma rápida e dinâmica, pois cada um contribui com uma ideia sobre o tema e evita que poucos falem muito e alguns não falem nada.

A pergunta utilizada foi "qual(is) elemento(s) precisam ser incluído(s) na Instalação?". A seguir cada um explicou porque incluir tal(is) elemento(s). Ainda em círculo de cultura, definiu-se em qual cenário formaram-se tais elementos. Os participantes, individualmente ou em pequenos grupos, se responsabilizaram por levar os elementos indicados para compor a Instalação, organizada em uma sala de aula do Departamento de Solos. A medida que o cenário foi sendo construído, adaptações foram feitas, mas de forma geral respeitou-se o acordo coletivo.

A Instalação foi composta por seis cenas, sendo a central com resíduos e organismos formadores do solo (Figura 1a); outra com sementes diversas (Figura 1b); uma com croquis de comunidades rurais e fotos de jovens agricultores(as); uma com solos da região (Figura 1c); uma com fluxos de entradas e saídas de agroecossistemas do município de Viçosa (Figura 1d); uma com materiais sobre o efeito dos agrotóxicos em peixes e; uma com um simulador de erosão. Estas cenas foram dispostas de forma a permitir que as pessoas pudessem caminhar pela Instalação.

Panos cobriram a maioria dos "tesouros" (cenas da Instalação e seus componentes), para que os participantes pudessem "descobrir" os "tesouros" de acordo com suas próprias percepções e curiosidades.

A Instalação se iniciou com uma dinâmica de abertura onde os participantes foram organizados em duplas, cada dupla teve cinco minutos para conhecer seu parceiro. Sugeriu-se que cada um perguntasse para o outro, pelo menos o nome, o local de origem e a expectativa com a Instalação. Em seguida, cada um se apresentou como se fosse seu parceiro. Após a apresentação, os participantes
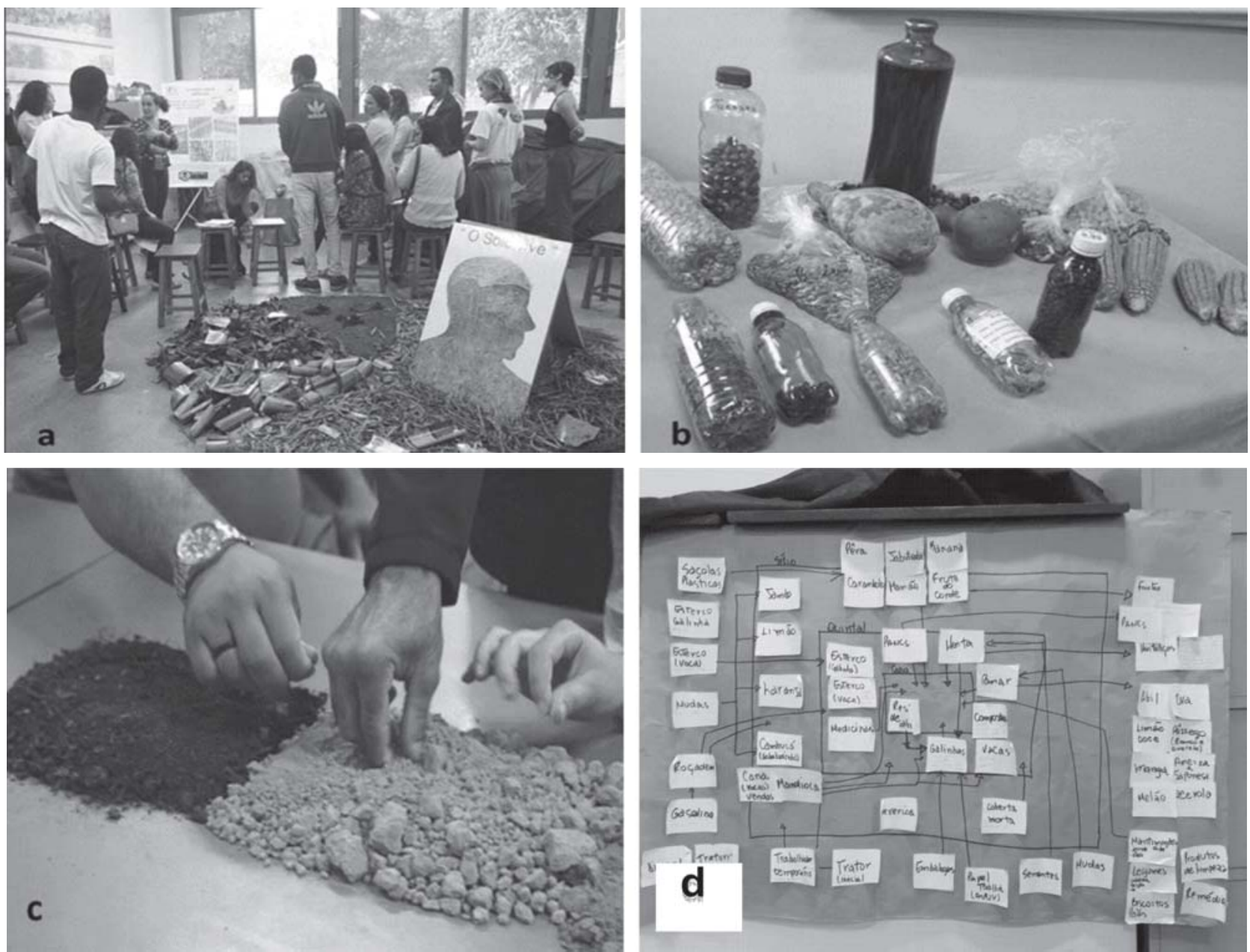

Figura 1 - Cenas da Instalação Artístico Pedagógica denominada “Tesouros escondidos da roça”, a) materiais vegetais e organismos do solo; b) sementes crioulas e transgênicas; c) solos predominantes na Zona da Mata Mineira e; d) fluxos de componentes presentes em comunidades rurais de Viçosa.

Fonte: A. Miranda e N. Casas 
indicaram qual "tesouro" deveria ser revelado primeiro, de forma aleatória, já que não podiam vê-lo. Após destampar a cena, os participantes foram incentivados a falar sobre o que estavam observando. Os facilitadores fizeram perguntas para problematizar o tema ou falas complementares antes de passar para a descoberta do próximo "tesouro" escondido.

Ao final, cada participante recebeu um pedaço de cartolina (tarjeta) para registrar uma resposta à pergunta "o que mais sensibilizou você durante a Instalação?". Cada participante escreveu uma palavra ou ideia que respondesse à pergunta. Através do círculo de cultura, cada um fez uma breve explicação da sua palavra ou ideia e a tarjeta foi disposta em uma cartolina tamanho A3, de forma a construir agrupamentos das principais ideias, fazendo as relações entre elas.

Para encerrar, cada participante, em uma avaliação simples, respondeu se sua expectativa havia sido alcançada. Uma mística de encerramento foi proposta, quando então um participante se sentiu motivado e convidou todos a rezarem um Pai Nosso. Por fim, alimentos agroecológicos e da roça, sementes e materiais didáticos, como cartilhas foram partilhadas.

\section{Resultados e Discussão}

Participaram da instalação 25 pessoas, a maioria mulheres e de origem rural. A metade dos participantes relacionavam-se com a área da educação, sendo oito jovens rurais estudantes, principalmente do curso de Licenciatura em Educação do Campo (LICENA) da UFV e duas professoras de escolas de Ensino Fundamental de Goiânia (GO). Dentre os estudantes do LICENA, uma era surda. Para atuar como interprete da estudante, participaram três profissionais em LIBRAS. Participaram também um casal de agricultores agroecológicos de Espera Feliz (MG) e duas crianças de aproximadamente dez anos. Os demais participantes foram os facilitadores da Instalação.

O título dado à Instalação atraiu muitos participantes para a atividade. Alguns participantes expressaram que gostaram do título da Instalação porque o nome falava da "roça" e isso tinha a ver com eles. Os objetos organizados na sala criaram um colash de cores e texturas, suscetíveis de serem tocados e até cheirados, o que permitiu explorar outros sentidos e não apenas a escuta. Isto possibilitou a inclusão e manifestação de todos, em especial das crianças e da jovem surda. Todos, mesmo com tanta diversidade, participaram efetivamente da Instalação, graças à metodologia utilizada.

A metodologia da instalação artístico pedagógica oportunizou a fala de todos(as) e permitiu a interação entre os participantes, o aprendizado coletivo e a construção dos saberes, a partir do diálogo entre gerações e experiências diversas. O círculo de cultura promoveu o compartilhamento de diferentes experiências e permitiu construir consensos a respeito do tema da Instalação.

A curiosidade dos participantes motivou a busca pelos tesouros, para, com surpresa, descobrirem que se tratavam de processos e organismos familiares a muitos deles e, em alguns casos, os tesouros eram os próprios participantes. Uma senhora, descobriu, entre as fotos, um tesouro importante: sua filha, que, coincidentemente, estava naquele dia fazendo 10 anos, assim como a Troca de Saberes.

Os conhecimentos tradicionais, muitas vezes invisibilizados, se revelaram como os grandes tesouros da roça. Recuperar e reconhecer os saberes tradicionais contribui para a autoestima e para a autonomia das pessoas do campo (LEFF, 2002). Desvelar tais conhecimentos é importante para o melhor entendimento sobre os sistemas de produção e para construir sistemas agroalimentares, agroecológicos e sustentáveis (GLIESSMAN, 2015).

Durante a Instalação, surgiram também importantes questões e possibilidades de pesquisa, como por exemplo, o estudo do conhecimento popular para indicar a contaminação de peixes por agrotóxicos. Com isto, aponta-se a Instalação Artístico-Pedagógica como um instrumento capaz de construir a indissociabilidade entre ensino, pesquisa e extensão, um princípio constitucional e indicado pelo Fórum de Pró-reitores (FORPROEX, 2012).

A troca de conhecimentos foi evidenciada na avaliação feita por alguns participantes, fornecendo como resultado comentários como: "a discussão sobre os solos e o manejo para produção me fez recordar conhecimentos que há tempos eu não o ativava e percebi que não sabia nada de terra quando vi aquelas agricultoras (inclusive crianças), falando de procedimentos naturais e eficientes de cultivo". A construção estética da instalação também foi avaliada positivamente. Segundo um participante, "[...] deu pra perceber o cuidado e carinho na preparação dessa instalação".

Os alimentos agroecológicos e da roça, trouxeram a discussão sobre a importância de compreender a alimentação como um ato político e relaciona-la com a saúde. Ainda, permitiu rapidamente discutir 
a necessidade de evitar o uso de descartáveis. A distribuição de sementes propiciou diálogos sobre a importância delas para a autonomia dos agricultores.

A mística foi proposta, por fazer parte da construção da identidade coletiva e tem sido muito utilizada pelos movimentos sociais e agroecológicos. No Movimento dos Trabalhadores Rurais Sem Terra (MST), a mística é considerada como uma forma de educação popular, uma prática pedagógica, cultural e política (COELHO, 2014; NASCIMENTO; MARTINS, 2008), uma forma de manter a identidade e ensinar a história através da evocação, do imaginário, do simbolismo, do que desencadeia mais facilmente os sentimentos e a afetividade (DAL RI; VIEITEZ, 2004). A oração do Pai Nosso, sugerida por um dos participantes, mesmo em uma sala de aula de uma universidade laica, é pertinente dentro da proposta pedagógica da Instalação. Ele traz a crença, subjetividade, sentimento e identidade daquele participante, considerados importantes na educação popular que se procura com a metodologia das instalações.

\section{Conclusões}

A Instalação Artístico-Pedagógica conseguiu estabelecer um diálogo amoroso e de forma horizontal entre os participantes e o entrelaçamento entre a sabedoria popular e o conhecimento científico. O círculo de cultura permitiu a contribuição de todos tanto no planejamento, quanto na realização da Instalação.

Os tesouros escondidos da roça foram revelados, a partir do próprio conhecimento dos participantes, que se sentiram (alguns deles) como parte dos tesouros. Estes tesouros são importantes na construção de sistemas alimentares agroecológicos.

\section{Agradecimentos}

Os autores do trabalho agradecem ao Núcleo de Agroecologia e Educação do Campo que junto com os parceiros e apoio da UFV constrói o evento "Troca de Saberes"; a todos os participantes e facilitadores da Instalação Artístico-Pedagógica "Os tesouros escondidos da roça", organizada como parte da X Troca de Saberes.

\section{Referências}

COELHO, Fabiano. A alma do MST? a praìtica da miìstica e a luta pela terra. Dourados-MS: Ed. UFGD, 2014. 290 p.

CONTE, G. M.; ZAFERINO, J.C.; OLIVEIRA, R.P.; BARBOSA, W.A. Terreiro Cultural: saberes populares como vivências educativas. IX Encontro Internacional do Fórum Paulo Freire, 2014. Anais [...] Centro de Referência Paulo Freire. Disponível em: http://www.acervo.paulofreire.org:8080/jspui/ handle/7891/3478 Acesso em: nov. 2018

DAL RI, N. M.; VIEITEZ, C. G. A educac'aPo do Movimento dos Sem-Terra: Instituto de Educac'aPo Josueì de Castro. Educ. Soc., Campinas, vol. 25, n. 89, p. 1379-1402, Set./Dez. 2004.

FÓRUM DE PRÓ-REITORES DE EXTENSÃO DAS UNIVERSIDADES PÚBLICAS BRASILEIRAS FORPROEX. Política Nacional de Extensão Universitária. Manaus, 2012.

FREIRE, P. Pedagogia da Autonomia: saberes necessários à prática educativa. São Paulo: Paz e Terra, 2011.

FREIRE, P. Pedagogia do oprimido. 42. ed. Rio de Janeiro: Paz e Terra; 2005.

GADOTTI, M. Saber aprender: um olhar sobre Paulo Freire e as perspectivas atuais da educação. Um olhar sobre Paulo Freire, Congresso Internacional, 20 a 23 de setembro de 2000. Anais [...] Centro de Referência Paulo Freire. Disponível em: http://acervo.paulofreire.org:8080/xmlui/handle/ 7891/1125 Acesso em: nov. 2018

GLIESSMAN, S. R. Agroecology: a global movement for food security and sovereignty. In: Agroecology for Food Security and Nutrition Proceedings of the FAO International Symposium Anais [...], 1819 de setembro 2014, Roma, Italia. FAO, 2015. 
LEFF, E. Agroecologia e Saber Ambiental. Agroecol.e Desenv.Rur.Sustent. Porto Alegre, v.3, n.1, jan./ mar. 2002.

NASCIMENTO, C. G.; MARTINS, L. C. Pedagogia da miìstica: as experiencias do MST. Emancipac'abo, Ponta Grossa, 8(2): 109-120, 2008.

SANTOS, M. L.; BARBOSA, W. A.; KÖLLN, M. Programa de extensão TEIA/UFV: formação universitária para uma ecologia de saberes. Educação em Revista, Belo Horizonte, v.29, n.04 | p.69-98, 2013.

VILLAR, J. P. et al. Troca de saberes-construindo diálogos entre conhecimento científico e saber popular. Cadernos de Agroecologia, v. 6, n. 2, 2011.

Submetido em: 30/10/2018 Aceito em: 03/05/2019. 